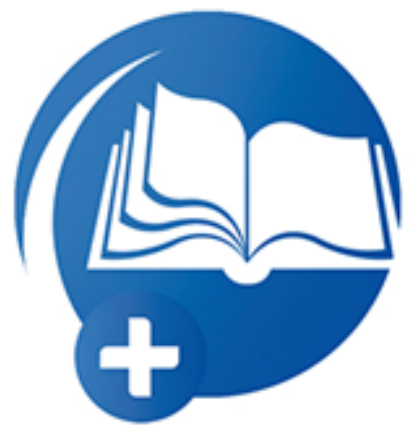

\title{
Parceria ensino-serviço na formação em odontologia
}

\author{
Sandra de Cássia Oliveira Barbosa ${ }^{1}$ \\ Maria de Fatima Nunes ${ }^{2}$
}

\begin{abstract}
RESUMO
O objetivo deste estudo foi sistematizar e compreender problemas diagnosticados nas unidades básicas de saúde da família (ESF), por estudantes da graduação em Odontologia de uma universidade pública. Foi utilizada a pesquisa documental para levantamento dos dados em projetos acadêmicos elaborados no período de 2006 a 2013, por alunos de graduação do sétimo período no Estágio em Odontologia Coletiva I. Os problemas relatados nos projetos acadêmicos aconteceram em 16 unidades de ESF e foram diagnosticados pelo método da estimativa rápida. Os 80 projetos elaborados pelos acadêmicos foram examinados e apresentou um total de 950 problemas diagnosticados. Os problemas elencados variaram de acordo com o território onde o estágio aconteceu. Após a coleta realizada pelos acadêmicos os problemas foram hierarquizados em função da viabilidade de intervenção. Os problemas priorizados foram aqueles que tiveram soluções a nível local. Após a coleta dos dados, foi realizada a categorização e emergiram sete categorias e 15 subcategorias. Dentre as categorias, as mais citadas foram 'gestão' e 'problema social' e das subcategorias foram 'gestão pública', 'gestão dos serviços de saúde' e 'organização dos processos de trabalho'. As categorias menos citadas foram 'trabalho em equipe' e 'controle social'. Concluiu-se que as categorias se interligam umas às outras, demonstrando que os problemas de saúde muitas vezes têm diversas causas e necessitam de soluções compartilhadas. Vários destes problemas não têm solução a nível local, relacionam-se aos Determinantes Sociais de Saúde e merecem atenção especial da gestão dos serviços. A categorização dos problemas demonstrou a amplitude das dificuldades vivenciadas pelas equipes de saúde da família.
\end{abstract}

Palavras chaves: Ensino na saúde. Ensino-serviço. Odontologia.

\section{Teaching-service partnership in dentistry training}

\begin{abstract}
The objective of this study was to systematize and understand problems diagnosed in basic family health units (FHS), by undergraduate students in Dentistry from a public university. Documentary research was used to collect data on academic projects prepared from 2006 to 2013, by undergraduate students from the seventh period in the Internship in Collective Dentistry I. The problems reported in academic projects took place in 16 basic family health units and were diagnosed by the rapid estimation method. The 80 projects prepared by the academics were examined and presented a total of 950 diagnosed problems. The problems listed varied according to the territory where the internship took place. After the collection carried out by the students, the problems were ranked according to the feasibility of the intervention. The prioritized problems were those that had solutions at the local level. After data collection, categorization was performed and seven categories and 15 subcategories emerged. Among

\footnotetext{
${ }^{1}$ Mestre em Ensino na saúde. Cirurgiã dentista da Secretaria Municipal de Saúde de Goiânia. E-mail: sandradecob@yahoo.com

2 Docente da Faculdade de Odontologia da Universidade Federal de Goiânia e do Mestrado Profissional no Ensino da Saúde.e-mail: maria_fatima_nunes@ufg.br
} 


\section{Programa de Pós-Graduação

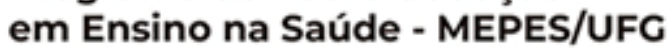 \\ DOSSIÊ \\ ENSINO NA SAÚDE}

V.17, N.01, 2021. ISSN: 1807-9342

the categories, the most cited were 'management' and 'social problem' and the subcategories were 'public management', 'health service management' and 'organization of work processes'. The least cited categories were 'teamwork' and 'social control'. It was concluded that the categories are interconnected to each other, demonstrating that health problems often have different causes and need shared solutions. Several of these problems have no solution at the local level, are related to social determinants of health and deserve special attention from the management of services. The categorization of the problems demonstrated the extent of the difficulties experienced by the family health teams.

Keywords: Teaching in health. Teaching-service. Dentistry.

\section{INTRODUÇÃO}

A Constituição Federal (BRASIl,1988) e a Lei Orgânica da Saúde (BRASIL, 1990a) estabelecem como competência do Sistema Único de Saúde (SUS), participar da formação de recursos humanos para a saúde e a produção de conhecimento na área.

A Portaria GM/MS nº 198 de 2004 (BRASIL, 2004a), emitida pelo Ministério da Saúde e alterada pela Portaria GM/MS nº 1.996 de 2007 (BRASIL, 2007a) instituíram a Política Nacional de Educação Permanente em Saúde (PNEPS) considerando a responsabilidade constitucional do Ministério da Saúde de ordenar a formação de recursos humanos para a área de saúde e de incrementar o desenvolvimento técnico-científico. Estas determinam que as universidades, pautadas nas diretrizes curriculares nacionais (DCN) (BRASIL, 2002), devem transformar, juntamente com os serviços, suas práticas de saúde e de ensino.

O papel de constatar a realidade e de produzir sentidos, no caso da saúde, pertence tanto ao SUS como às instituições formadoras de suas profissões. Cabe ao SUS e às instituições formadoras coletar, sistematizar, analisar e interpretar permanentemente informações da realidade, problematizar o trabalho e as organizações de saúde e de ensino e construir significados e práticas com orientação social, mediante participação ativa dos gestores setoriais, formadores, usuários e estudantes (CECCIM; FEUERWERKER, 2004).

O conhecimento da realidade e os problemas existentes em regiões onde os estágios acontecem são utilizados para as ações acadêmicas e contribuem para distanciar o acadêmico do paradigma cartesiano, que ainda se faz muito presente nos cursos de graduação na área da saúde. E essa parceria ensino-serviço também contribui para a educação permanente da equipe 


\section{Programa de Pós-Graduação

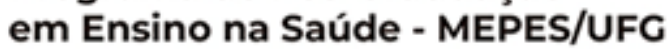 \\ DOSSIÊ \\ ENSINO NA SAÚDE}

V.17, N.01, 2021. ISSN: 1807-9342

de saúde da família e os dados disponibilizados podem, inclusive, servir para planejamento da equipe e gestão local.

A vivência da realidade do serviço não se encerra apenas com o aprendizado de competências e habilidades de ordem técnica, mas favorece o conhecimento e entendimento dos determinantes sociais existentes no território da equipe de saúde da família e desenvolve no acadêmico um olhar ampliado do processo saúde-doença (BRASIL, 2007a; BULGARELLI et al., 2014; SALES et al., 2015; MOREIRA et al., 2019).

Os estágios acadêmicos estão sujeitos à Lei no 11.788/2008 da Presidência da República e regulamentados nas instituições formadoras (BRASIL, 2008).

Na Faculdade de Odontologia da UFG (FO/UFG), o Estágio em Odontologia Coletiva I (EOC I) utiliza-se do planejamento em saúde para problematizar a realidade da Estratégia Saúde da Família (ESF). Em qualquer formato de planejamento em saúde, uma das etapas é o diagnóstico e análise dos problemas existentes e seus condicionantes de saúde.

Problema de saúde pode ser definido como algo considerado fora dos padrões de
normalidade para o ator social ${ }^{3}$ que está analisando uma determinada situação, no que
se refere aos riscos à saúde, às formas de adoecimento e morte da população
(problemas do estado de saúde da população) e à organização e funcionamento do
sistema de saúde (problemas do sistema de saúde) (VILLASBÔAS, 2004, p.10).

Desde o início do processo com a observação da realidade para detectar os possíveis problemas existentes, até a intervenção para solucioná-los, os alunos são levados a se inserirem nesta realidade para entenderem o contexto da situação na busca de soluções satisfatórias.

Desde que o Estágio em Odontologia Coletiva I se estabeleceu na ESF, em 2004 (NUNES, 2014) não se realizou nenhum levantamento e análise dos problemas diagnosticados nos vários anos. Os resultados poderão ser úteis como subsídios de planejamento da gestão municipal e/ou local, além de ampliar a discussão no meio acadêmico no sentido de que os

\footnotetext{
${ }^{3}$ Ator social é toda pessoa, instituição pública ou privada, grupos sociais organizados e o Estado, que têm poder para influenciar uma determinada situação (VILASBÔAS, 2004).
} 


\section{Programa de Pós-Graduação \\ em Ensino na Saúde - MEPES/UFG \\ DOSSIÊ \\ ENSINO NA SAÚDE}

ITINERARIUS

REFLECIONIS

V.17, N.01, 2021. ISSN: 1807-9342

produtos gerados em estágios curriculares no SUS possam subsidiar discussões e aproveitamento de seus dados.

Os trabalhos existentes sobre estágios, em geral são relatos de experiências ou pesquisas sobre percepções de profissionais ou acadêmicos, sem o envolvimento da comunidade (BULGARELLI et al., 2014; SALES et al., 2015; MOREIRA et al., 2019). Cabe ao SUS e às instituições formadoras coletar, sistematizar, analisar e interpretar permanentemente informações da realidade, problematizar o trabalho e as organizações de saúde e de ensino, além de construir significados e práticas com orientação social, mediante participação ativa dos gestores setoriais, formadores, usuários e estudantes (CECCIM; FEUERWERKER, 2004, p. 46).

Diante destas reflexões, o questionamento que norteou este estudo foi: quais os problemas diagnosticados pelos acadêmicos de EOC I da FO/UFG nas unidades da ESF no município de Goiânia no período de 2006 a 2013? A hipótese era de que os problemas mais comuns a todas as unidades da ESF seriam de competência da gestão, seguidos de aspectos relacionados aos condicionantes sociais de saúde.

\section{METODOLOGIA}

O presente estudo é uma pesquisa documental com dados primários extraídos de 80 (oitenta) projetos elaborados por acadêmicos do sétimo período do curso de Odontologia da Universidade Federal de Goiás (FO/UFG), na disciplina de Odontologia Coletiva que utilizava o planejamento em saúde para problematizar a realidade de equipes que integravam a saúde da família. Os dados obtidos neste estudo são relativos aos problemas diagnosticados e hierarquizados durante o estágio com vistas a selecionar um problema passível de intervenção. Problematizar "é refletir sobre determinadas situações, questionando fatos, fenômenos e ideias, compreendendo os processos e propondo soluções" (BRASIL, 2005).

Os estágios da EOC I eram realizados por grupos compostos de quatro a seis alunos sob supervisão de um(a) professor(a) e um(a) cirurgiã(o) dentista preceptor. O objetivo deste 


\section{Programa de Pós-Graduação

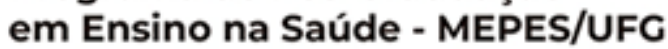 \\ DOSSIÊ \\ ENSINO NA SAÚDE}

V.17, N.01, 2021. ISSN: 1807-9342

estágio era "analisar a saúde geral e bucal como um processo, com ênfase nos determinantes sociais e planejar a sua atuação junto a grupos populacionais" (NUNES et al., 2014).

\subsection{Critérios de inclusão e exclusão}

Como critério de inclusão dos problemas na análise documental é que estivessem em projetos escritos no período de 2006 a 2013. O período foi definido pela razão de que os projetos neste período utilizaram a mesma metodologia de planejamento e, consequentemente, a forma de diagnosticar e apresentar os problemas do território.

Como critério de exclusão foram retirados os problemas que se repetiram nos diversos projetos pois não foi considerado a quantidade de vezes que um determinado problema tenha sido apresentado. Também foram excluídos aqueles que não se apresentassem em forma inteligível.

\subsection{Coleta e análise dos dados}

Os projetos foram coletados de caixas-arquivo depositadas na FO/UFG, no período pesquisado e estavam nos formatos impresso e/ou digital. Do item "hierarquização dos problemas" de cada projeto foi realizada a coleta dos dados por meio da leitura dos projetos e registros dos problemas em uma planilha Excel.

Após a transcrição e análise dos dados fez-se uma primeira categorização dos problemas por uma das pesquisadoras, servidora da Secretaria Municipal de Saúde, e para melhor confiabilidade dos dados foi realizada nova checagem e categorização pela mesma pesquisadora e uma docente do referido estágio. A categorização se deu pela similaridade entre os problemas dentro do contexto da saúde. Em seguida as categorias foram também quantificadas na busca de um melhor entendimento sobre sua ocorrência, visando compreender os vários aspectos relacionados.

Depois de criadas e após várias leituras das categorias observou-se a necessidade de criar subcategorias de acordo com a especificidade de cada um dos problemas. Em seguida foi feita análise de conteúdo temática e os problemas categorizados foram observados à luz da 


\section{Programa de Pós-Graduação

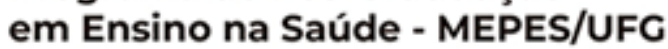 \\ DOSSIÊ \\ ENSINO NA SAÚDE}

V.17, N.01, 2021. ISSN: 1807-9342

literatura e de sua contribuição para a formação dos envolvidos nesse processo no período pesquisado.

\subsection{Aspectos ético-legais}

Após a obtenção da anuência da equipe de docentes do estágio e do Conselho Diretor da FO/UFG, o projeto foi submetido ao Comitê de Ética em Pesquisa da UFG, por meio da Plataforma Brasil tendo parecer favorável no 705.304 de 30/06/2014.

\section{RESULTADOS E DISCUSSÃO}

Do total de 80 (oitenta) projetos formulados pelos acadêmicos para as unidades básicas de saúde da família foram analisados 950 (novecentos e cinquenta) problemas diagnosticados.

Os problemas elencados pelos estudantes tinham similaridades e variações de acordo com o território e o grupo de estagiários. As variações existentes nos problemas de uma mesma unidade de saúde pode ter ocorrido por razões, como a equipe da ESF não ser a mesma no período estudado e isto pode ter influenciado na percepção da realidade; as microáreas analisadas não foram as mesmas, mesmo sendo a mesma unidade de saúde; os estagiários podem ter apresentado um olhar diferenciado para observar a realidade; os métodos utilizados para coleta dos problemas permitiram observações da realidade e interpretações diferentes de um ano para o outro e alguns problemas podem ter sidos solucionados e outros podem ter surgidos.

Neste contexto, uma mesma situação pode representar um problema para uma pessoa, enquanto para outra nada representa. Isto acontece porque um indivíduo se interessa pela situação e encontra dificuldades para resolvê-la, enquanto outro não a percebe ou não se interessa, ou ainda, possui mecanismos para resolvê-la (SANTOS et al., 2013).

Em todo o período analisado, o estágio ocorreu em dezesseis (16) Unidades Básicas de Saúde da Família distribuídas em quatro dos sete Distritos Sanitários da Secretaria Municipal de Saúde (SMS), o que proporcionou aos estudantes a observação de diferentes territórios com suas especificidades em relação aos determinantes sociais e a organização dos serviços. Por 


\section{Programa de Pós-Graduação

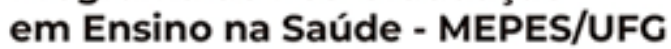 \\ DOSSIÊ \\ ENSINO NA SAÚDE}

V.17, N.01, 2021. ISSN: 1807-9342

outro lado, a preceptoria do cirurgião dentista da equipe era exercida voluntariamente, o que se supõe que era conduzida com envolvimento e comprometimento do profissional. Todos foram aspectos importantes para a vivência dos estudantes no SUS e aproximação do modelo de atenção da ESF.

Do período de 2006 a 2011, os problemas detectados pelos estudantes, na região onde aconteceu o estágio, foram obtidos a partir do método da estimativa rápida. A estimativa rápida é bastante utilizada em diagnósticos para os planejamentos em saúde, pois coleta informações fundamentalmente em três fontes de dados: registros escritos, entrevistas com informantes chaves e observação de campo (ACÚRCIO; SANTOS; FERREIRA, 1998).

Nos anos de 2012 e 2013, os estudantes também listaram os problemas a partir da observação na área de abrangência da unidade de saúde e na coleta de dados documentais, mas sem seguir um roteiro pré-determinado como no método da estimativa rápida.

Durante o estágio todos os problemas percebidos foram relacionados e em seguida hierarquizados, levando-se em consideração a viabilidade de resolução e, por fim, definido o problema que receberiam intervenção. Os problemas priorizados que receberam intervenções pelos estudantes foram aqueles que tiveram soluções no nível local dos serviços de saúde, como por exemplo, a elaboração de mapa vivo das microáreas assistidas pela equipe.

\subsection{Categorização dos problemas}

$\mathrm{Na}$ categorização, emergiram sete categorias e quinze subcategorias dos problemas elencados no estudo (Quadro 1). 


\section{Programa de Pós-Graduação

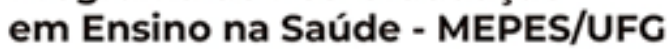 \\ DOSSIÊ \\ ENSINO NA SAÚDE}

V.17, N.01, 2021. ISSN: 1807-9342

Quadro 1. Categorias e subcategorias dos problemas diagnosticados por acadêmicos de odontologia em estágio no SUS. Goiânia, 2006-2013.

\begin{tabular}{|ll|}
\hline \multicolumn{1}{|c|}{ Categorias } & \multicolumn{1}{c|}{ Subcategorias } \\
\hline Gestão & Gestão pública \\
& Gestão dos serviços de saúde \\
\hline Saúde & Saúde geral \\
& Saúde bucal \\
\hline & Afetivo \\
& Educação formal e informal \\
& Apoio social \\
Social & Serviços sociais \\
& Condições de vida e trabalho \\
& Drogas lícitas e ilícitas \\
& Violência e criminalidade \\
& Equipe \\
& Profissionais de outros serviços \\
& População \\
\hline Informação & \\
& Não emergiram subcategorias \\
\hline Trabalho em equipe & \\
\hline Controle social & \\
\hline Organizacional &
\end{tabular}

Fonte: BARBOSA; NUNES, 2020.

As categorias emergiram percentualmente em relação aos novecentos e cinquenta (950) problemas elencados. Quinhentos e setenta (60\%) dos problemas estavam nas categorias 'gestão' e 'social'. Destes, 314 (33\%) eram da categoria 'gestão' e 256 (27\%) pertenciam à categoria 'social' (Gráfico 1). Os problemas destas categorias dependem de grandes intervenções, vontade política, parcerias intersetoriais e planejamento conjunto, além de geralmente, demandar um tempo grande para sua completa ou parcial resolução. Estes aspectos determinam a não governabilidade para intervenção por acadêmicos em estágio. 


\section{Programa de Pós-Graduação \\ em Ensino na Saúde - MEPES/UFG \\ DOSSIÊ \\ ENSINO NA SAÚDE}

ITINERARIUS

REFLECIONIS

V.17, N.01, 2021. ISSN: 1807-9342

Gráfico 1. Números absolutos e relativos das categorias de problemas relatados nos projetos de acadêmicos de odontologia em estágio no SUS. Goiânia, 2006-2013.

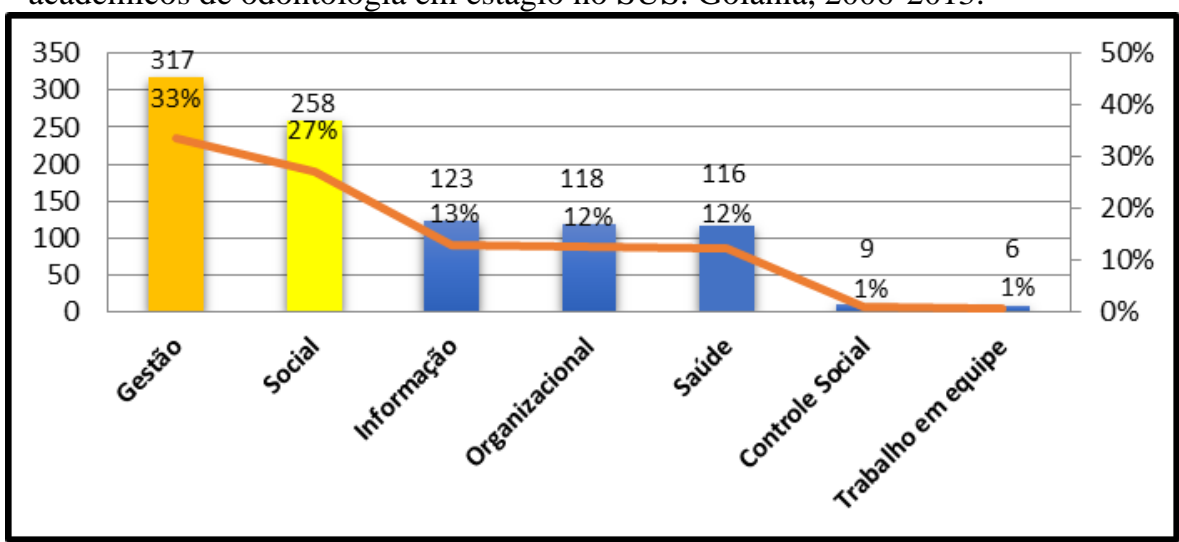

Fonte: BARBOSA; NUNES, 2020

Em relação aos 380 (40\%) dos demais problemas detectados, 123 (13\%) emergiram as categorias 'informação', 116 (12\%) a categoria 'saúde' e 118 (12\%) a categoria 'organizacional'. Todas elas tiveram praticamente a mesma representatividade entre 2006 e 2013, dentro do cenário geral das categorias (Gráfico1).

Gráfico 2. Números absolutos e relativos das subcategorias de problemas relatados nos projetos por acadêmicos de odontologia em estágio no SUS. Goiânia, 2006-2013.

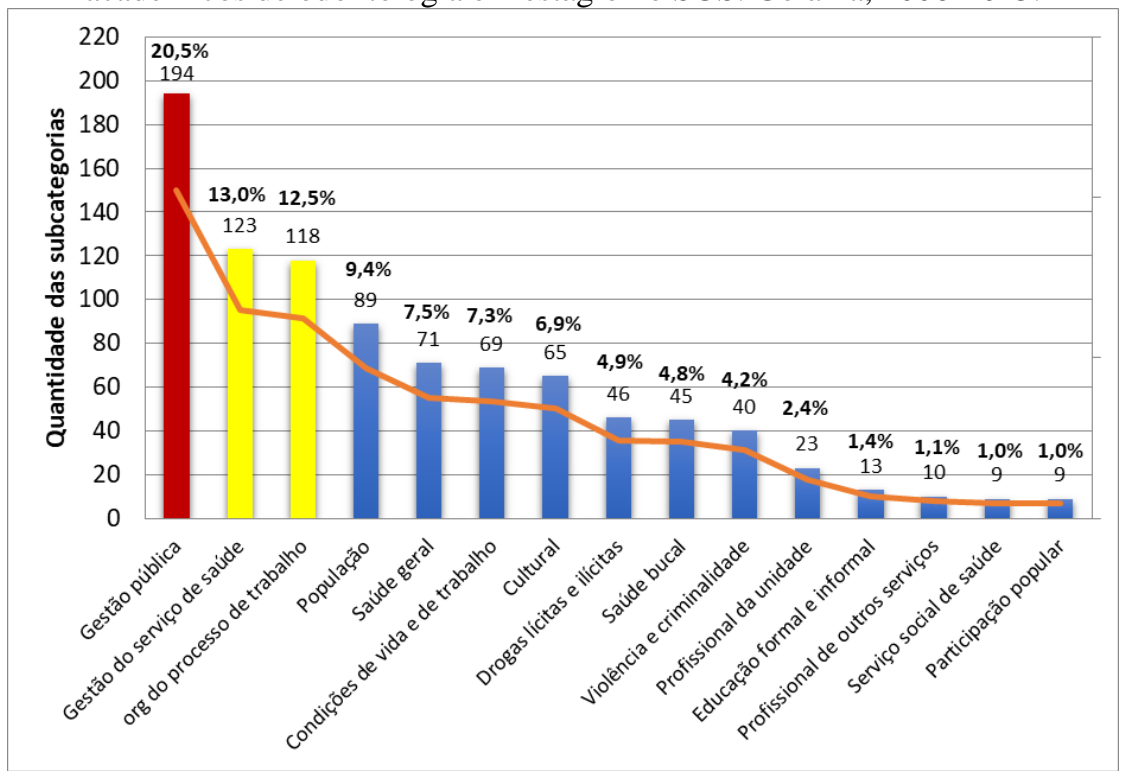

Fonte: BARBOSA; NUNES, 2020. 


\section{Programa de Pós-Graduação \\ em Ensino na Saúde - MEPES/UFG \\ DOSSIÊ \\ ENSINO NA SAÚDE}

ITINERARIUS

REFLECIONIS

V.17, N.01, 2021. ISSN: 1807-9342

Quanto às subcategorias (Gráfico 2), observa-se que as mais representativas ao longo dos anos foram 'gestão pública', que foram citadas em 194 problemas (20,5\%), 'gestão do serviço de saúde', que apresentou 123 problemas (13\%) e 'organização do processo de trabalho' $(118 ; 12,5 \%)$. Todas estas subcategorias estão relacionadas a algum tipo de gestão, o que aponta ser a mesma um grande entrave para a atenção adequada à população. Da gestão depende em grande parte a eficiência dos serviços de saúde.

\section{Categoria Gestão}

Esta foi a categoria mais citada nos trabalhos em estudo. É interessante o fato de que aspectos da gestão foram percebidos em todos os grupos do estágio dos acadêmicos de odontologia. As competências e habilidades gerais presentes nas Diretrizes Curriculares dos cursos de graduação da área de saúde contemplam a administração e gerenciamento tornandose imprescindível a compreensão das condições e dos elementos gerais de gestão em saúde e gestão em serviços (ARAKAWA, 2013).

Os problemas desta categoria se arrastaram ao longo do período sem uma solução definitiva (Gráfico 3). Todos estes fatores contribuem para a necessidade de reformulação no processo de trabalho. Alguns desses problemas se esbarram na questão do financiamento ou da gestão desses recursos (OCKE-REIS, 2008; SALDIVA, VERAS, 2018; FUNCIA, 2019).

Ao longo dos anos, a "categoria Gestão" foi mais percebida: saiu de 29\% para 51\% do total de problemas encontrados. É uma porcentagem considerável e que leva à necessidade de reflexão por parte da gestão do município (Gráfico 3).

Gráfico 3. Evolução da categoria 'gestão' ao longo dos anos de estágio no SUS. Goiânia, 2006-2013.

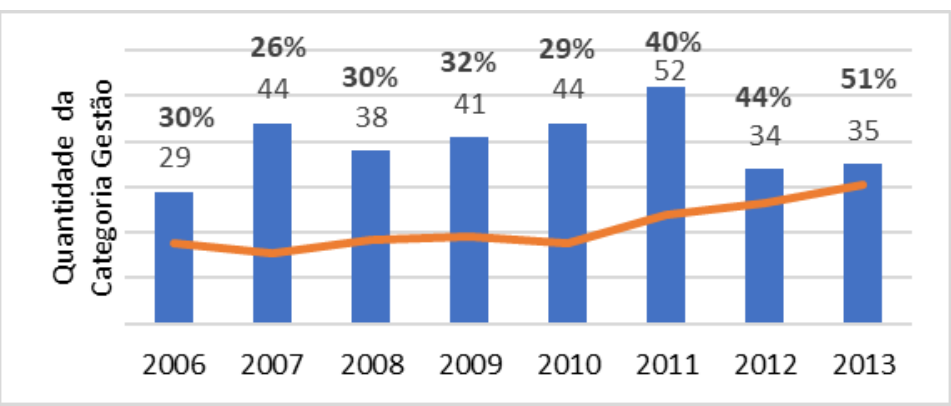

Fonte: BARBOSA; NUNES, 2020. 


\section{Programa de Pós-Graduação \\ em Ensino na Saúde - MEPES/UFG \\ DOSSIÊ \\ ENSINO NA SAÚDE}

ITINERARIUS

REFLECIONIS

V.17, N.01, 2021. ISSN: 1807-9342

A categoria "Gestão" estava constituída pelas subcategorias 'Gestão pública' e 'Gestão dos serviços de saúde'.

Ao longo dos anos, a 'gestão pública' significou grande parte dos problemas relacionados à 'gestão' detectados pelos acadêmicos em estágio, principalmente em 2011, representando 85\% do total dos problemas desta categoria. Porém, em 2013 observa-se uma inversão, sendo a gestão do serviço de saúde mais representativa. Não é possível afirmar as causas dessa ocorrência, pois elas não foram investigadas, mas o fato de ter ocorrido mudança na metodologia utilizada para a coleta dos dados, pode ser uma influência a ser considerada (Gráfico 4).

Gráfico 4. Subcategorias 'gestão dos serviços de saúde' e 'gestão pública' detectadas como problemas por acadêmicos de Odontologia em unidades básicas de saúde do SUS. Goiânia, 2006-2013.

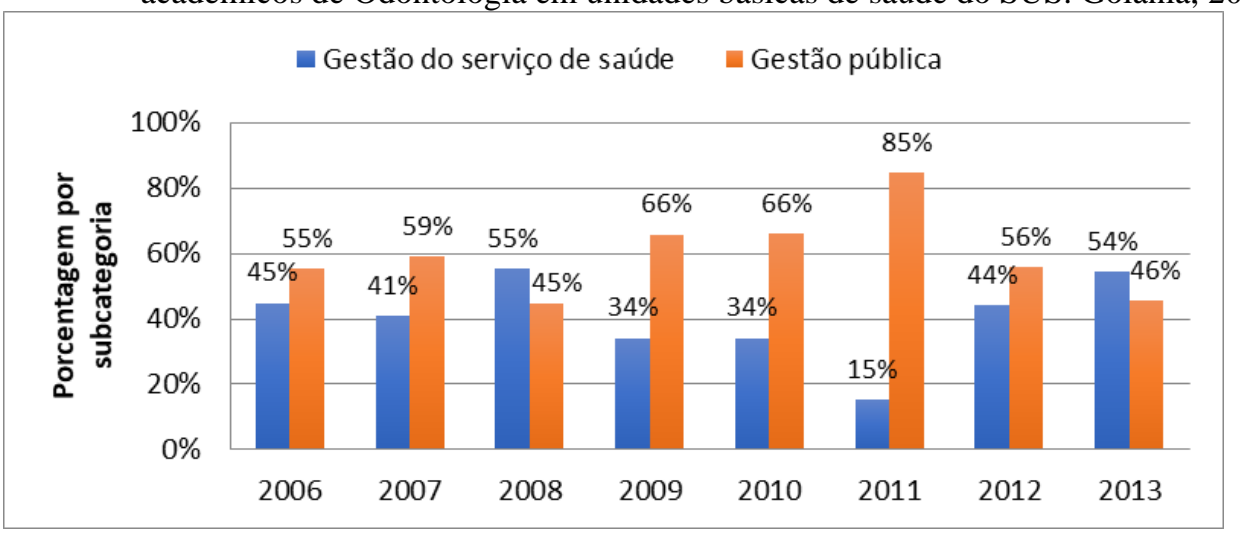

Fonte: BARBOSA; NUNES, 2020.

O Ministério da Saúde do Brasil, por intermédio da Secretaria de Gestão Estratégica e Participativa implantou, em 2007, o ParticipaSUS - Política Nacional de Gestão Estratégica e Participativa no SUS (BRASIL, 2009). O ParticipaSUS “constitui-se em um conjunto de atividades voltadas ao aprimoramento da gestão do SUS, visando maior eficácia, eficiência e efetividade" (BEZERRA et al, 2012). Entretanto, sendo o financiamento um gargalo para a gestão, seja em nível nacional, estadual ou municipal, este foi extremamente comprometido pela aprovação da Emenda Constitucional do teto de gastos públicos, que congela o financiamento da saúde e da educação por vinte anos (BRASIL, 2016; VIEIRA; PIOLA, 2016; MONTEIRO, 2017; MARIANO, 2017). 


\section{Programa de Pós-Graduação

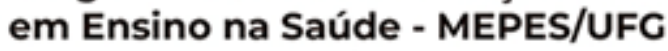 \\ DOSSIÊ \\ ENSINO NA SAÚDE}

ITINERARIUS

REFLECIONIS

V.17, N.01, 2021. ISSN: 1807-9342

Sobre a 'Gestão dos serviços de saúde', os problemas ocorridos nesta subcategoria se caracterizaram principalmente pela fragmentação e complexidade de seus problemas. Ramos e Rosa (2016) afirmaram que "a organização do processo de trabalho não funciona se for hierarquizado, centralizador e prescritivo". Alguns dos problemas citados: falta de carro para todos os integrantes da equipe; falta de funcionamento dos equipamentos odontológicos; falta de materiais de consumo; fichas de cadastro inacessíveis para as equipes de saúde bucal; ausência de gestor na unidade. Alguns desses problemas necessitarem de solução compartilhada com outros setores, outros necessitam somente da reorganização do serviço.

A 'Gestão dos serviços de saúde' inclui a gestão de recursos humanos e muitas vezes constitui um entrave para os serviços de saúde, o que dificulta sua operacionalização. A necessidade de habilidades definidas, assim como técnicas e práticas que consigam administrar comportamentos e potencializar as habilidades de cada profissional é um desafio presente na rotina do trabalho. O desempenho dos profissionais do serviço de saúde depende também da qualidade do que é oferecido para realizar seu trabalho e à satisfação daqueles que utilizam dos serviços prestados.

O número insuficiente de Agente Comunitário de Saúde (ACS) nas equipes, chama a atenção devido ao fato de aparecer diversas vezes ao longo de todo período pesquisado. Foram relatados problemas como: falta de grande número de ACS; microáreas descobertas de ACS. Estes problemas inviabilizam o que é preconizado para o serviço realizado pelas equipes da ESF e dificultam a compreensão por parte dos acadêmicos estagiários sobre a real composição das equipes de saúde e até mesmo de conhecerem todos os desafios das microáreas ou realizarem visitas domiciliares.

Os problemas relacionados à 'gestão dos serviços de saúde', em sua maioria, comprometem o acesso do usuário ao serviço. Trata-se de desafio para o que se espera em relação ao cuidado integral. Tem importância significativa para a gestão, inclusive constitui em indicador relevante para a avaliação dos serviços de saúde (SOUZA et al, 2008). 


\section{Programa de Pós-Graduação

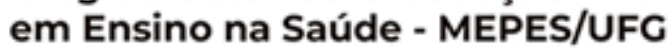 \\ DOSSIÊ \\ ENSINO NA SAÚDE}

V.17, N.01, 2021. ISSN: 1807-9342

\section{Categoria Social}

Os problemas desta categoria relacionam-se com Determinantes Sociais de Saúde (DSS) que são diversos, variados e interferem diretamente no estado de saúde das pessoas e comunidades. Estão relacionados com fatores individuais e coletivos. Esta categoria é formada por sete (7) subcategorias (Gráfico 5), que emergiram de acordo com a natureza dos problemas:

- 'Subcategoria afetivo'. Problemas que se relacionam com o aspecto emocional das pessoas como: solidão dos idosos; baixa autoestima, carência afetiva.

- Subcategoria 'educação formal e informal'. Incorpora as questões relacionadas à educação de forma geral, como: ausência de escola e cursos profissionalizantes na região; baixa escolaridade; falta de conhecimento sobre reciclagem de materiais.

- $\quad \mathrm{Na}$ 'subcategoria cultural'. Os problemas relacionam-se com hábitos ou atitudes culturais das pessoas como: desvalorização da prevenção odontológica e higiene geral; lixos depositados em locais inadequados, gravidez na adolescência.

- Subcategoria 'apoio social'. Problemas que dificultam a inserção social. Existem problemas como: falta de ocupação e entretenimento para os jovens; falta de inserção social do idoso; dificuldades de acesso dos idosos à unidade de saúde; falta de informação e inclusão para os idosos. 


\section{Programa de Pós-Graduação}

em Ensino na Saúde - MEPES/UFG

\section{Dossiê.}

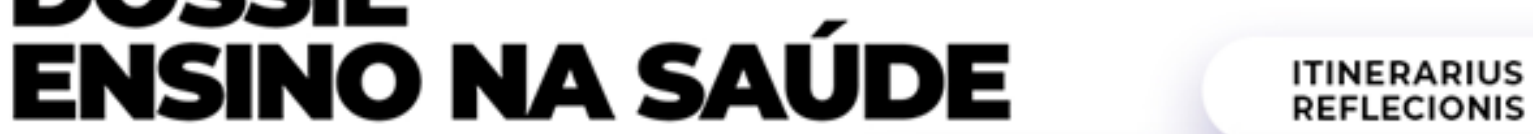

\section{V.17, N.01, 2021. ISSN: 1807-9342}

Gráfico 5. Representatividade das subcategorias da "categoria social", por ano de estágio dos acadêmicos de Odontol ogia em unidades básicas de saúde do SUS. Goiânia, 2006-2013.

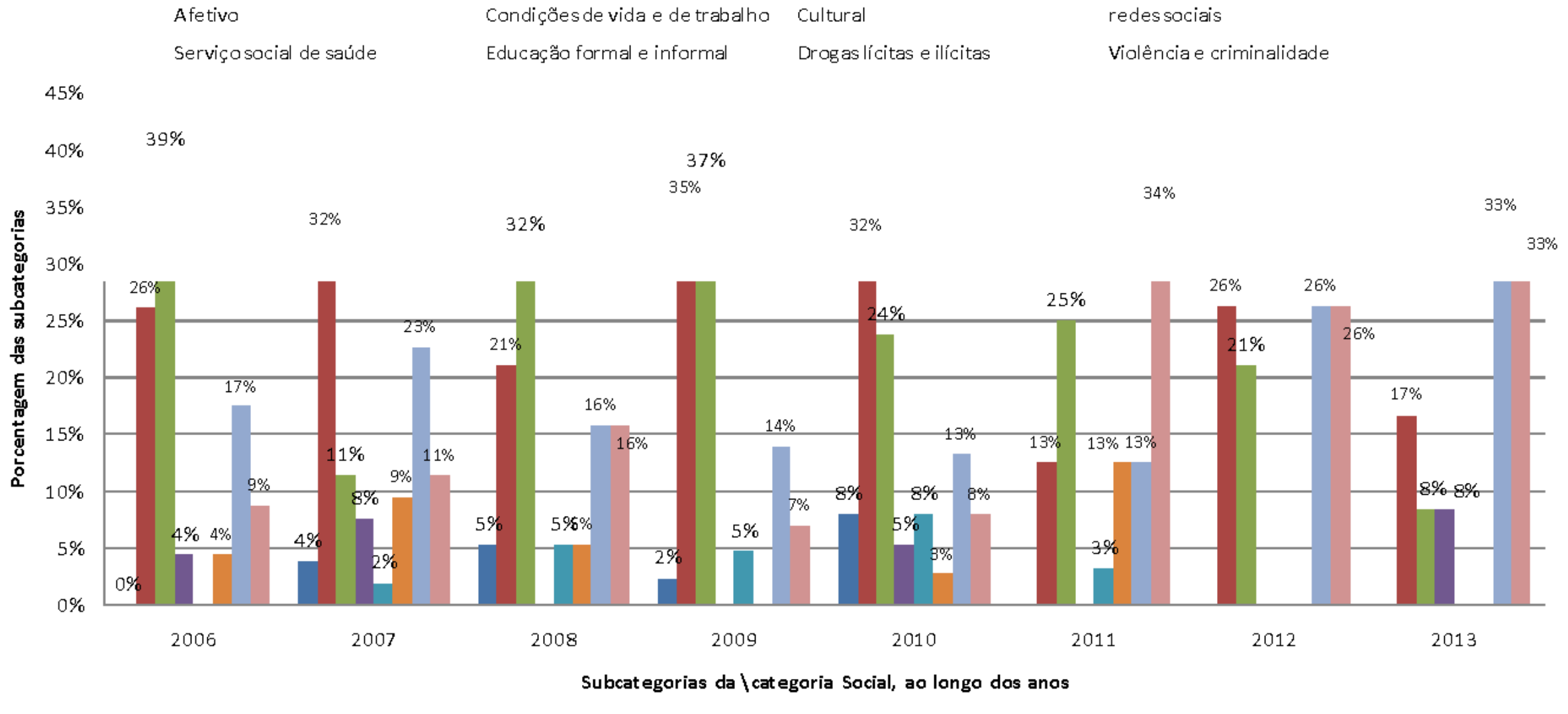

Fonte: BARBOSA; NUNES, 2020. 


\section{Programa de Pós-Graduação \\ em Ensino na Saúde - MEPES/UFG \\ DOSSIÊ \\ ENSINO NA SAÚDE}

- $\quad$ Subcategoria 'redes sociais'. Os problemas relacionam-se com a falta de instituições sociais que privam as pessoas de benefícios como: falta de aparelhos sociais; falta de Centro Municipal de Educação Infantil (CMEI).

- Subcategoria 'condições de vida e trabalho'. Problemas como: presença de população flutuante; inversão dos valores quanto ao consumo de bens materiais; desemprego.

- Subcategoria 'drogas lícitas e ilícitas'. Encontram-se aqui problemas como: grande número de usuários de drogas criando pontos de venda e consumo; alto índice de alcoolismo e tabagismo.

- Subcategoria 'violência e criminalidade'. Nesta subcategoria incluem problemas como: abuso sexual; grande número de assaltos; tráfico de drogas.

- Subcategoria 'condições de vida e trabalho'. Problemas como: presença de população flutuante; inversão dos valores quanto ao consumo de bens materiais; desemprego; prostituição.

- Subcategoria 'drogas lícitas e ilícitas'. Encontram-se aqui problemas como: grande número de usuários de drogas criando pontos de venda e consumo; alto índice de alcoolismo e tabagismo.

- Subcategoria 'violência e criminalidade'. Nesta subcategoria incluem problemas como: abuso sexual; grande número de assaltos; tráfico de drogas.

Alguns problemas poderiam estar em diferentes subcategorias como, por exemplo, tráfico de drogas que também poderia ser incluído na subcategoria 'drogas lícitas e ilícitas'. Para melhor discernir foi necessário observar o contexto da citação do problema.

As subcategorias encontradas na 'categoria social' foram relacionadas de acordo com dos Determinantes Sociais de Saúde (BUSS; PELLEGRINI FILHO, 2007), que permite uma visualização das relações hierárquicas entre os diversos determinantes da saúde e que estão diretamente relacionados com as condições de vida e saúde das pessoas.

Constatações relevantes em relação aos DSS referem-se à de pertencer a grupos sociais excluídos da maioria dos benefícios da sociedade, como as relatadas nos problemas 'falta de 


\section{Programa de Pós-Graduação

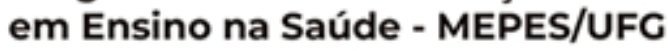 \\ DOSSIÊ \\ ENSINO NA SAÚDE}

ITINERARIUS

REFLECIONIS

V.17, N.01, 2021. ISSN: 1807-9342

escola na região', 'falta de cursos profissionalizantes', 'desemprego', dentre outros. Esta percepção causa constrangimentos, discriminação e sentimentos de inferioridade que refletem na determinação das condições de saúde das pessoas (OMS, 2011).

Há também os fatores psicossociais baseados nas relações entre as desigualdades sociais como 'população flutuante', 'consumo de drogas”, os mecanismos psicobiológicos e a situação de saúde como no caso de 'grande número de depressão entre os idosos', “a única renda da família é a aposentadoria da avó', 'residências de dois cômodos para várias pessoas'. Buss; Pellegrini Filho (2007) afirmaram que o estresse causado pela vivência em sociedades desiguais provoca prejuízos consideráveis para a saúde.

Para enfrentar os problemas causados por DSS é preciso realizar ações coerentes e coordenadas entre si que envolvam os setores da sociedade, públicos e privados, como trabalhar com ações continuadas realizadas por longos períodos de tempo como buscar soluções para 'assaltos no bairro', 'tráfico de drogas', 'alta taxa de desemprego'; 'uso de drogas lícitas e ilícitas', realização equitativa de práticas que promovam bem-estar incentivadas tanto por iniciativas públicas quanto privadas ‘adequações de áreas para prática de lazer e esporte' e atuar na conscientização da sociedade quanto a importância da realização destas ações (OMS, 2011).

As DCN para Odontologia orientam que a formação do cirurgião dentista deve contemplar e atender as necessidades sociais de saúde, com ênfase no SUS (BRASIL, 2002).

As atividades realizadas durante a formação acadêmica, devem proporcionar aos alunos oportunidades para que tenham novos olhares para as necessidades de saúde dos sujeitos, que contribuam para a formação de profissionais de saúde mais qualificados e preocupados com suas responsabilidades sociais (SANTOS et al., 2013).

Em relação aos problemas de cunho social, há uma grande alternância entre as subcategorias ao longo do período analisado. As subcategorias 'condições de vida e trabalho' e 'cultural' aparecem de forma significativa até 2012. Outro ponto de atenção relaciona-se à 'violência e criminalidade', que apesar de aparecerem em todos os anos do período analisado, a partir de 2011 aparecem com porcentagens expressivas. 


\section{Programa de Pós-Graduação

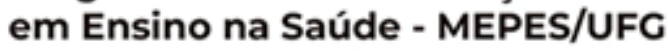 \\ DOSSIÊ \\ ENSINO NA SAÚDE}

\section{Categoria Saúde}

A “categoria saúde" é constituída pelas subcategorias 'saúde geral' e 'saúde bucal'. Baseou-se nos modelos explicativos do processo saúde/doença que interferem na condição de saúde individual e coletiva da população.

Essa, contrariamente ao que podia se esperar, não obteve um resultado quantitativamente representativo como as categorias 'gestão' e 'social' presentes no Gráfico 1. Este resultado pode estar ligado ao fato de que na maioria dos anos utilizou-se a metodologia da estimativa rápida para o diagnóstico da realidade. Esta metodologia considera todos os determinantes socias da saúde. Também pode ter outros fatores que interferiram, como a falta de ACS para coletar as informações pertencentes à área adscrita.

A 'saúde bucal' teve grande representatividade (75\%) dentro dos problemas relacionados à saúde, apenas em 2006 (Gráfico 6). Nos anos seguintes, a 'saúde geral' foi apontada como problema principal, na grande maioria dos casos, chegando a $83 \%$ do total de problemas relacionados à saúde no ano de 2011. Dentre esses problemas os mais citados estão os relacionados com hipertensão, diabetes, obesidade, depressão.

Gráfico 6. Subcategorias 'saúde bucal' e 'saúde geral' da "categoria saúde” por anos de estágio no SUS dos acadêmicos de odontologia. Goiânia, 2006 - 2013.

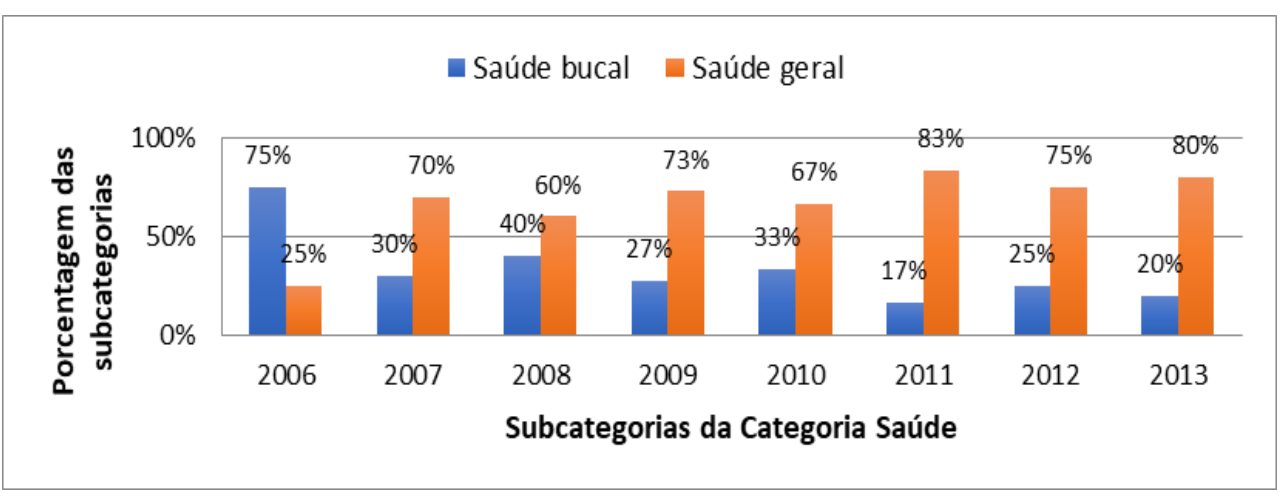

Fonte: BARBOSA; NUNES, 2020.

Em muitos casos as causas de saúde geral e bucal relacionam-se com fatores comuns de risco, necessitando de intervenções compartilhadas com profissionais de outras categorias profissionais, para sua completa resolução. A necessidade de mensurar o impacto que estes 


\section{Programa de Pós-Graduação

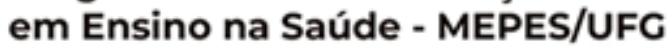 \\ DOSSIÊ \\ ENSINO NA SAÚDE}

ITINERARIUS

REFLECIONIS

V.17, N.01, 2021. ISSN: 1807-9342

agravos causam nas pessoas contribui para o planejamento de ações de promoção da saúde e prevenção de doenças (GOES et al., 2012).

\section{Categoria Informação}

Dos $100 \%$ dos problemas analisados, $13 \%$ deles se relacionam com a categoria informação (Gráfico 7).

Gráfico 7. Subcategorias 'população', 'profissional da unidade', 'profissional de outros serviços', 'outros profissionais' da "categoria informação". Goiânia, 2006-2013.

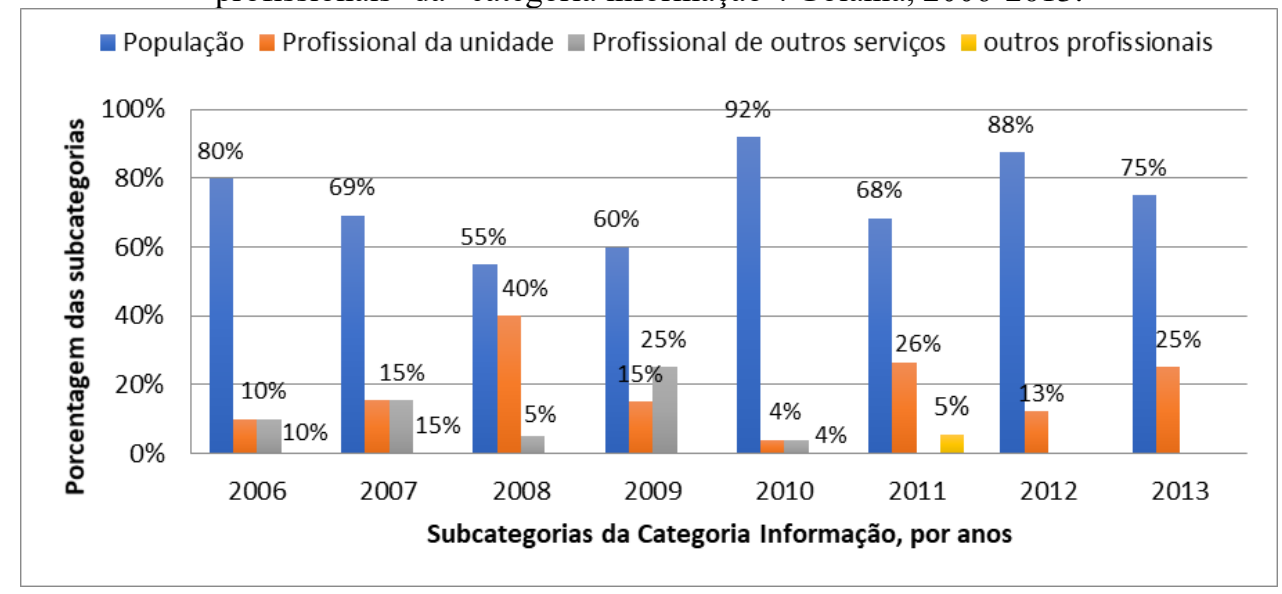

Fonte: BARBOSA; NUNES, 2020.

Um grande número que referia "dificuldades em coletar informações". Os problemas relativos ao sistema de informação no SUS, são decisivos para a tomada de decisões da gestão. Sem informações adequadas há grande dificuldades de planejar as ações de saúde. Nesta categoria foram listados também os problemas como "desconhecimento da comunidade sobre o trabalho da ESF e o serviço oferecido pela unidade"; "falta de comunicação entre os membros das equipes"; "profissionais de odontologia que não se integram".

\section{Categoria Trabalho em equipe}

A integração de profissionais para realizarem atividades, demonstra maturidade para o trabalho e satisfação dos usuários. Além disto, a participação em reuniões e a discussão de casos contribuem para o aprendizado profissional e a melhoria do trabalho oferecido (SANTANA, 


\section{Programa de Pós-Graduação

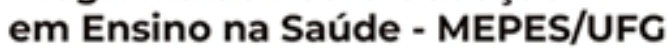 \\ DOSSIÊ \\ ENSINO NA SAÚDE}

ITINERARIUS

REFLECIONIS

V.17, N.01, 2021. ISSN: 1807-9342

2000; PERES et al., 2011). A realização do trabalho em equipe é condição fundamental para o desenvolvimento das ações propostas para a ESF. Considerando que a "comunicação é deficiente" entre os profissionais na "Categoria Informação', pode-se conjecturar que o problema foi negligenciado.

\section{Categoria Organizacional}

A organização dos serviços de saúde perpassa por todas as categorias analisadas, na busca de uma oferta de serviço que atenda as reais necessidades da população. 'Carência de fluxograma de atendimento mais organizado', 'grande número de famílias não cadastradas', 'falta de informações para a população sobre o trabalho realizado pela unidade', 'espera longa para procedimentos especializados' foram alguns dos problemas listados nesta categoria e que dificultam a organização do trabalho.

A falta de realização de atividades como as percebidas nos problemas, "necessidade da unidade em organizar a demanda para atendimento odontológico", "falta de programas e atendimentos a adultos (donas de casa)", "inexistência de atuação da ESF no aparelho social da microárea", "dificuldades para realizar atividades educativas fora da unidade de saúde", "preenchimento deficiente de ficha", impedem uma organização eficiente do serviço realizado pelas equipes (AGUILAR-DA-SILVA; SCAPIN; BATISTA, 2011).

\section{Categoria Controle social}

Desde a criação da lei 8.142 (BRASIL, 1990b) o controle social deve participar das decisões relativas à saúde da população nas três esferas de governo: federal, estadual e municipal. A partir de então a participação popular nas decisões referentes à saúde é essencial, pois os participantes dos conselhos vivenciam os problemas e compartilham da mesma realidade da população assistida (ROLIM et al., 2013). De acordo com os problemas relatados, percebeu-se que a população ainda não entendeu a importância de sua participação nas decisões de saúde. A maioria dos problemas se relacionou com a inexistência dos conselhos locais de saúde, ou à falta de atuação de seus conselheiros. 


\section{Programa de Pós-Graduação

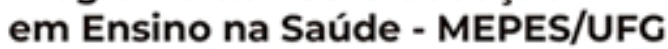 \\ DOSSIÊ \\ ENSINO NA SAÚDE}

V.17, N.01, 2021. ISSN: 1807-9342

É através da participação efetiva da população que acontece o fortalecimento dos conselhos de saúde para atuarem na reivindicação e fiscalização das ações de saúde, com o olhar focado na integralidade das ações e universalidade do acesso aos usuários de maneira equânime (CECCIM; FEUERWERKER, 2004).

\section{CONCLUSÕES}

Concluiu-se que as categorias se interligam umas às outras, demonstrando que os problemas de saúde muitas vezes têm diversas causas e necessitam de soluções compartilhadas. Vários destes problemas não têm solução a nível local, relacionam-se aos DSS e merecem atenção especial da gestão dos serviços. A categorização dos problemas demonstrou a amplitude das dificuldades vivenciadas pelas equipes de saúde da família.

\section{REFERÊNCIAS}

ACÚRCIO, Francisco de Assis; SANTOS, Marcos André dos; FERREIRA, Sibele M. Gonçalves. O planejamento local de serviços de saúde. In: MENDES, Eugênio Vilaça (org). A organização de saúde no nível local. São Paulo: Hucitec; Cap. 3; p.111-32.1998.

AGUILAR-DA-SILVA, R. H.; SCAPIN, L. T.; BATISTA, N. A. Avaliação da formação interprofissional no ensino superior em saúde: aspectos da colaboração e do trabalho em equipe. Avaliação (Campinas), Sorocaba, v.16, n.1, p.165-184, Mar. 2011. Disponível em: https://www.scielo.br/pdf/aval/v16n1/v16, n1-9. Acesso em: 26 de mar. 2020. https://doi.org/10.1590/S1414-40772011000100009.

ARAKAWA, Aline Megumi et al. Gestão em saúde: o aprendizado e a formação acadêmica de estudantes de graduação. Revista CEFAC, São Paulo, v. 15, n. 4, p. 947-956, Aug. 2013. Disponível em: https://www.scielo.br/pdf/rcefac/v15n4/23.pdf. Acesso em: 26 de mar. 2020. https://doi.org/10.1590/S1516-18462013000400024.

BEZERRA, Luciana Caroline de Albuquerque et al. Identificação e caracterização dos elementos constituintes de uma intervenção: pré-avaliação da política ParticipaSUS. Ciênc. saúde coletiva, Rio de Janeiro, v.17, n.4, p.883-900, Apr. 2012. Disponível em: <http://www.scielo.br/scielo.php?script=sci_arttext\&pid=S1413$81232012000400011 \& \operatorname{lng}=e n \& n r m=i s o>$. Acesso em 27 de mar. 2020. https://doi.org/10.1590/S1413-81232012000400011. 


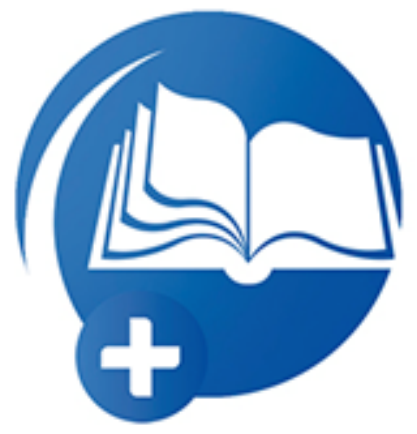

\section{Programa de Pós-Graduação

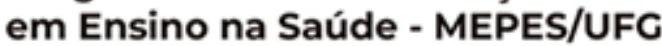 \\ DOSSIÊ \\ ENSINO NA SAÚDE}

BRASIL. Constituição da República Federativa do Brasil. Constituição (1988). Brasília. Disponível em: http://www.planalto.gov.br/ccivil_03/Constituicao/Constituicao.htm. Acesso em: 26 mar. 2020.

BRASIL. Lei $\mathbf{n}^{\mathbf{0}}$ 11.788, de 25 de setembro de 2008. Dispõe sobre o estágio de estudantes e dá outras providências. Disponível em: http://www.planalto.gov.br/ccivil_03/_ato20072010/2008/lei/111788.htm. Acesso em: 29 de mar. 2020.

BRASIL. Ministério da Educação e Cultura. CNE. CES. Resolução CNE/CES de 3 de fevereiro de 2002. Institui Diretrizes Curriculares Nacionais do Curso de Graduação em Odontologia. Diário Oficial da União, Brasília, 4 mar. Seção 1. P. 10. 2002. Disponível em: http://portal.mec.gov.br/cne/arquivos/pdf/CES032002.pdf. Acesso em: 26 de mar. 2020.

BRASIL. Ministério da Saúde. Secretaria de Gestão Estratégica e Participativa. Política Nacional de Gestão Estratégica e Participativa no SUS - ParticipaSUS / Ministério da Saúde, Secretaria de Gestão Estratégica e Participativa. - 2. ed. - Brasília: Editora do Ministério da Saúde, 2009. 44 p. - (Série B. Textos Básicos de Saúde) ISBN 978-85-334-1485-3. Disponível em:

http://bvsms.saude.gov.br/bvs/publicacoes/politica_estrategica_participasus_2ed.pdf. Acesso em 27 de mar. 2020.

BRASIL. Diário Oficial da República Federativa do Brasil Lei n. 8.080, de 19 de dezembro de 1990. Brasília, 19 dez 1990, seção 1, p. 18055. Dispõe sobre as condições para a promoção, proteção e recuperação da saúde, a organização e o funcionamento dos serviços correspondentes e dá outras providências. 19 dez 1990a. Disponível em: http://www.planalto.gov.br/ccivil_03/leis/18080.htm. Acesso em: 31 de mar. 2020.

BRASIL. Presidência da República. Casa Civil. Subchefia para Assuntos Jurídicos. Lei no 8.142, de 28 de dezembro de 1990. Brasília, 1990b. Dispõe sobre a participação da comunidade na gestão do Sistema Único de Saúde (SUS) e sobre as transferências intergovernamentais de recursos financeiros na área da saúde e dá outras providências.

Disponível em: http://www.planalto.gov.br/ccivil_03/leis/L8142.htm. Acesso em: 30 mar. 2020.

BRASIL. Presidência da República. Casa Civil. Subchefia para Assuntos Jurídicos. Emenda Constitucional $n^{0}$ 95, de 15 de dezembro de 2016. Altera o Ato das Disposições Constitucionais Transitórias, para instituir o Novo Regime Fiscal, e dá outras providências. Disponível em: http://www.planalto.gov.br/ccivil_03/constituicao/Emendas/Emc/emc95.htm. Acesso em 26/03/2020.

BRASIL. Ministério da Saúde. Secretaria de Gestão do Trabalho e da Educação na Saúde. Departamento de Gestão da Educação na Saúde. A Educação Permanente entra na roda: Polos de educação permanente em saúde: conceitos e caminhos a percorrer. Brasília: 


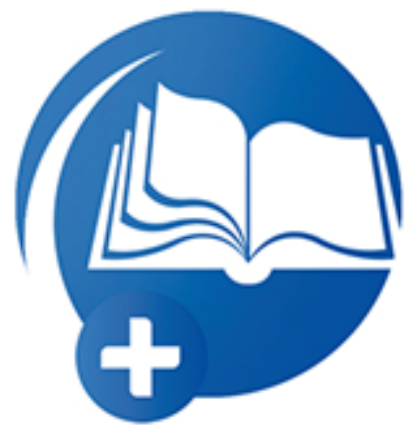

\section{Programa de Pós-Graduação \\ em Ensino na Saúde - MEPES/UFG \\ DOSSIÊ \\ ENSINO NA SAÚDE}

ITINERARIUS

REFLECIONIS

V.17, N.01, 2021. ISSN: 1807-9342

Ministério da Saúde, 2005. 36 p.: il. color. - (Série C. Projetos, Programas e Relatórios) (Educação na Saúde) ISBN 85-334-0973-7. Disponível em:

http://bvsms.saude.gov.br/bvs/publicacoes/educacao_permanente_entra_na_roda.pdf. Acesso em 27 de mar. 2020. [ [ Links ]

BRASIL. Ministério da Saúde. Gabinete do ministro. Portaria n¹.996 de 20 de ago. 2007a. Dispõe sobre as diretrizes para a implementação da Política Nacional de Educação Permanente em Saúde. Disponível em:

https://bvsms.saude.gov.br/bvs/saudelegis/gm/2007/prt1996_20_08_2007.html. Acesso em: 26 de mar. 2020.

BUSS, P. M.; PELLEGRINI FILHO, A. A saúde e seus determinantes Sociais. Revista de Saúde Coletiva, Rio de Janeiro - RJ, v. 17, n. 1, p. 77- 93, 2007c. Disponível em: http://www.scielo.br/pdf/physis/v17n1/v17n1a06.pdf. Acesso em: 30 mar. 2020.

BULGARELLI, Alexandre Favero et al. Formação em saúde com vivência no Sistema Único de Saúde (SUS): percepções de estudantes do curso de Odontologia da Universidade Federal do Rio Grande do Sul (UFRGS), Brasil. Interface (Botucatu), Botucatu, v.18, n. 49, p.351362, June 2014. Available from <http://www.scielo.br/scielo.php?script=sci_arttext\&pid=S1414$32832014000200351 \& \operatorname{lng}=e n \& n r m=i s o>$. access on 20 Nov. 2020. Epub Mar 17, 2014. https://doi.org/10.1590/1807-57622013.0583.

CECCIM, R. B.; FEUERWERKER, L. C. M. Mudança na graduação das profissões de saúde sob o eixo da integralidade. Cadernos Saúde Pública, Rio de Janeiro , v. 20, n. 5, p. 14001410, Oct. 2004. Disponível em:.311X2004000500036\&lng=en\&nrm=iso>. Acesso em: 31 Mar. 2020. https://doi.org/10.1590/S0102-311X2004000500036.

BUSS, Paulo Marchiori; PELLEGRINI FILHO Alberto. A Saúde e seus Determinantes Sociais. PHYSIS: Rev. Saúde Coletiva, Rio de Janeiro, v.17, v.1, p.77-93, 2007. Disponível em: https://www.scielo.br/pdf/physis/v17n1/v17n1a06.pdf. Acesso em: 31 Mar. 2020.

FUNCIA, Francisco Rózsa. Subfinanciamento e orçamento federal do SUS: referências preliminares para a alocação adicional de recursos. Cienc Saúde Colet, Rio de Janeiro, v. 24, n. 12, p. 4405-4415, Dec. 2019. Available from <http://www.scielo.br/scielo.php?script=sci_arttext\&pid=S1413-

81232019001204405\&lng=en\&nrm=iso>. access on 01 Apr. 2020. Epub Nov 25, 2019. https://doi.org/10.1590/1413-812320182412.25892019.

GOES, Paulo Sávio Angueiras de et al. Vigilância à saúde bucal: a construção de um modelo integrado. Editorial. Cad. Saúde Pública, Rio de Janeiro, 28 Sup:S6-S7, 2012. Disponível em: https://www.scielo.br/pdf/csp/v28s0/02.pdf. Acesso em: 20 Nov.2020.

MARIANO, Cynara Monteiro. Emenda constitucional 95/2016 e o teto dos gastos públicos: Brasil de volta ao estado de exceção econômico e ao capitalismo do desastre. Rev. Investig. 


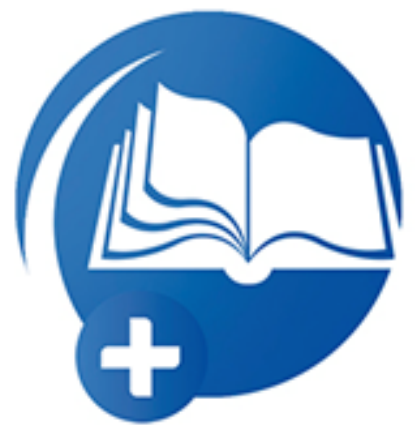

\section{Programa de Pós-Graduação \\ em Ensino na Saúde - MEPES/UFG \\ DOSSIÊ \\ ENSINO NA SAÚDE}

ITINERARIUS

REFLECIONIS

V.17, N.01, 2021. ISSN: 1807-9342

Const., Curitiba, v. 4, n.1, p. 259-281, Apr. 2017. Available from

$<$ http://www.scielo.br/scielo.php?script=sci_arttext\&pid=S2359-

$56392017000100259 \& \operatorname{lng}=\mathrm{en} \& n r m=\mathrm{iso}>$. access on 20 Nov.2020. Epub Apr 15,

2019. https://doi.org/10.5380/rinc.v4i1.50289.

MOREIRA Katia Fernanda Alves et al. Aprendendo, ensinando e mapeando território: vivências de acadêmicos de Enfermagem. Rev Eletron. Acervo Saúde, v. 11, n. 4, p. 1-8, 2019. Disponível em: https://acervomais.com.br/index.php/saude/article/view/240/164.

Acesso em: 22 Out.2020.

NUNES, Maria de Fátima; FREIRE, Maria do Carmo Matias; QUEIROZ, Maria Goretti; NOVAIS, Tatiana Oliveira; MARCELO, Vânia Cristina. Universidade Federal de Goiás. Faculdade de Odontologia.Estágio em Odontologia Coletiva I. GUIA DO ESTÁGIO EM ODONTOLOGIACOLETIVA I - 2014. 26p.

OCKE-REIS, C. O. Os problemas de gestão do SUS decorrem também da crise crônica de financiamento?. Trabalho, educação e saúde, Rio de Janeiro, v. 6, n. 3, p. 613-622, 2008 . Available from <http://www.scielo.br/scielo.php?script=sci_arttext\&pid=S1981$77462008000300012 \& \operatorname{lng}=$ en\&nrm=iso $>$. Access

on 29 Mar. 2020. https://doi.org/10.1590/S1981-77462008000300012.

ORGANIZAÇÃO MUNDIAL DA SAÚDE - OMS. Declaração Política do Rio sobre Determinantes Sociais da Saúde. Rio de Janeiro. 2011. Disponível em: http://www.who.int/sdhconference/discussion_paper/Discussion_Paper_PT.pdf acesso em $11 / 10 / 2020$.

SANTANA, José Paranaguá de ( org.). Organização do cuidado a partir de problemas: uma alternativa metodológica para a atuação da Equipe de Saúde da Família /colaboração do Ministério da Saúde e do Polo de Capacitação em Saúde da Família da UFMG: NESCON- Faculdade de Medicina e Escola de Enfermagem. — Brasília: Organização Pan-Americana da Saúde/ Representação do Brasil, 2000.

PERES, Cássia Regina Fernandes Biffe et al. O Agente Comunitário de Saúde frente ao processo de trabalho em equipe: facilidades e dificuldades. Revista da Escola de Enfermagem. USP, São Paulo - SP, v. 45, n. 4, p. 905 - 11, 2011. Disponível em: $<$ http://www.scielo.br/scielo.php?script=sci_arttext\&pid=S0080-

62342011000400016\&lng=en\&nrm=iso>. ISSN 0080-6234. https://doi.org/10.1590/S0080$\underline{62342011000400016 .}$

RAMOS, Lais Helena Fomingues; ROSA, Anderson da Silva. Planejamento e Gestão de Serviços de Saúde. UNA-SUS-Universidade Aberta do SUS. Especialização em saúde da família. Unidade de Conteúdo 12. Disponível em: https://www.unasus.unifesp.br/biblioteca_virtual/pab/7/unidades_conteudos/unidade12/unida de12.pdf. Acesso em 29 de mar. 2020. 


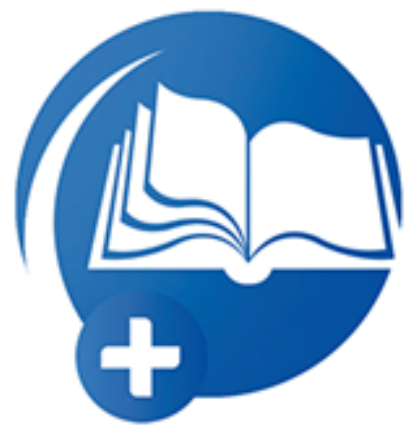

\section{Programa de Pós-Graduação \\ em Ensino na Saúde - MEPES/UFG \\ DOSSÎÉ NA SAÚDE}

ITINERARIUS

REFLECIONIS

V.17, N.01, 2021. ISSN: 1807-9342

ROLIM, Leonardo Barbosa; CRUZ, Rachel de Sá Barreto Luna Callou; SAMPAIO, Karla Jimena Araújo de Jesus. Participação popular e o controle social como diretriz do SUS: uma revisão narrativa. Saúde debate, Rio de Janeiro, v.37, n. 96, p.139-147, Mar.2013.Available from <http://www.scielo.br/scielo.php?script=sci_arttext\&pid=S0103-

$11042013000100016 \& \operatorname{lng}=$ en\&nrm $=$ iso $>$. access

on 21 Nov. 2020. https://doi.org/10.1590/S0103-11042013000100016.

SALES, Patrícia Regina de Souza; MARIN, Maria José Sanches; SILVA FILHO, Carlos Rodrigues da. INTEGRAÇÃO ACADEMIA-SERVIÇO NA FORMAÇÃO DE

ENFERMEIROS EM UM HOSPITAL DE ENSINO. Trab. educ. saúde, Rio de Janeiro, v. 13, n. 3, p. 675-693, Dec. 2015. Available from

<http://www.scielo.br/scielo.php?script=sci_arttext\&pid=S1981-

$77462015000300675 \& \operatorname{lng}=$ en\&nrm $=$ iso $>$. access

on 21 Nov. 2020. https://doi.org/10.1590/1981-7746-sip00057.

SALDIVA, Paulo Hilário Nascimento; VERAS, Mariana. Gastos públicos com saúde: breve histórico, situação atual e perspectivas futuras. Estudos avançados, São Paulo, v.32, n. 92, p. 47-61, Apr. 2018. Available from

<http://www.scielo.br/scielo.php?script=sci_arttext\&pid=S0103-

$40142018000100047 \& \operatorname{lng}=$ en $\& n r m=$ iso $>$. access

on 31 Mar. 2020. https://doi.org/10.5935/0103-4014.20180005.

SANTOS, Karina Tonini dos et al. Percepção discente sobre a influência de estágio extra muro na formação acadêmica odontológica. Revista odontológica da UNESP.

Araraquara, v.42, n.6, p.420-425, Dec. 2013. Available from

<http://www.scielo.br/scielo.php?script=sci_arttext\&pid=S1807-

$25772013000600005 \& \operatorname{lng}=$ en $\& n r m=i$ iso $>$. access

on 01 Apr. 2020. https://doi.org/10.1590/S1807-25772013000600005.

SOUZA, Elizabethe Cristina Fagundes de et al. Acesso e acolhimento na atenção básica: uma análise da percepção dos usuários e profissionais de saúde. Cad. de Saúde Pública, Rio de Janeiro, v. 24, supl. 1, p. s100-s110, 2008. Disponível em:

$<$ http://www.scielo.br/scielo.php?script=sci_arttext\&pid=S0102-

$311 \mathrm{X} 2008001300015 \& \operatorname{lng}=\mathrm{pt} \& \mathrm{nrm}=\mathrm{iso}>$. Acesso

em: 30 mar. 2020. https://doi.org/10.1590/S0102-311X2008001300015.

VIEIRA, Fabiola Sulpino; PIOLA, Sérgio Francisco. Instituto de Pesquisa Econômica e Aplicada- IPEA. TD 2260 - Implicações do Contingenciamento de Despesas do Ministério da Saúde para o Financiamento Federal do Sistema Único de Saúde. Instituto de Pesquisa Econômica e Aplicada- IPEA. TD 2260. Brasília, dez. de 2016. Disponível em:

http://www.ipea.gov.br/portal/index.php?option=com_content $\&$ view=article \&id=29199\&Ite $\underline{\text { mid=406. }}$. Acesso em 29 de mar. 2020. 
VILASBÔAS, A. L. Q. Planejamento e programação das ações de vigilânciada saúde no nível local do Sistema Único de Saúde. Rio de Janeiro: FIOCRUZ/EPJV/PROFORMAR, 2004c. 68p. 